A. Pramesh Rao, G. Swarup and Gopal-Krishna, eds.

\title{
Probing Large Scale Structures in HI with GMRT
}

\author{
S. Bharadwaj \\ Department of Physics \& Center for Theoretical Studies, I.I.T. \\ Kharagpur, 721302, India
}

B.B. Nath

Raman Research Institute, Bangalore 560080, India

S.K. Sethi

Mehta Research Institute, Chhatnag Road, Jhusi, Allahabad 211019, India

\begin{abstract}
The redshifted $1420 \mathrm{MHz}$ emission from the $\mathrm{HI}$ in unresolved Lyman- $\alpha$ clouds (DLA) at high $\mathrm{z}$ will appear as a background radiation in low frequency radio observations. We calculate the brightness temperature and the angular two-point correlation function of the fluctuations of this radiation. This can be an important probe of the large-scale structure at high $\mathrm{z}$.
\end{abstract}

\section{Introduction}

The density of HI $\left(\Omega_{\mathrm{HI}}\right)$ in DLAs has been determined reasonably well from absorption studies for $0 \lesssim z \lesssim 3$ (Lanzetta, Wolfe, \& Turnshek 1995). Although it has not been possible to observe an individual damped Lyman- $\alpha$ cloud in emission due to its small size, it is worthwhile to ask whether this emission can be detected as a background radiation. In addition these clouds, like the matter distribution at high $z$, are expected to be clustered, which will cause fluctuations in the background radiation. We investigate the possibility of using the fluctuations in the contribution from redshifted HI emission to the extragalactic background radiation at low radio frequencies (meter wave) to probe the large scale structure at high $z$. This is largely motivated by the fact that the Giant Meter-wave Radio Telescope, (GMRT, Swarup et al. 1991) which is designed to observe in several frequency bands in the interval $150 \mathrm{MHz}$ to $1420 \mathrm{MHz}$, has recently started functioning.

\section{Results}

We consider a spatially flat FRW cosmological model with $h=0.5$ and $\Omega_{m}=$ 1. For the matter power spectrum we use the analytic fitting form given by Efstathiou, Bond and White (1992) with $\Gamma=0.25$ normalized to $\sigma_{8}=0.6$. We also assume that the HI faithfully traced the matter distribution. The isotropic 


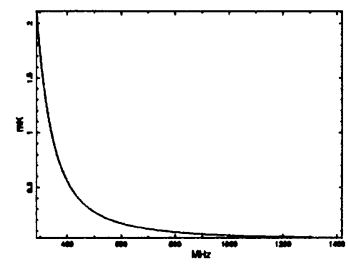

Figure 1. This shows the isotropic part of the background temperature at different frequencies.
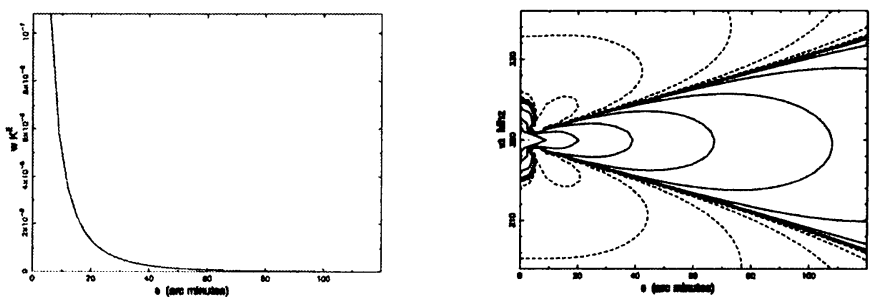

Figure 2. The first graph shows $w\left(\nu_{1}, \nu_{2}, \theta\right)$ in $\mathrm{K}^{2}$ vs. $\theta$ with $\nu_{1}=$ $\nu_{2}=320 \mathrm{Mhz}$. The second graph shows contours of equal $w\left(\nu_{1}, \nu_{2}, \theta\right)$ at logarithmic intervals of $w$ with $\nu_{1}=320 \mathrm{Mhz}$. Here $1^{\prime}=1.8 \mathrm{Mpc}$ and $1 \mathrm{Mhz}=4.6 \mathrm{Mpc}$. The dashed lines show negative values of $w$.

part of the background antenna temperature due to the HI in DLAs is shown in figure 1. We see that the background temperature at $\nu=327 \mathrm{MHz}$ (one of the channels of GMRT) is $\approx 1 \mathrm{mK}$.

The quantity we propose for analysing the fluctuation in the temperature is $w\left(\nu_{1}, \nu_{2}, \theta\right)$ - the cross-correlation between the temperature fluctuations at two different frequencies $\nu_{1}$ and $\nu_{2}$ along two lines of sight separated by an angle $\theta$. The fluctuation in the matter density and the peculiar velocities both contribute to the temperature fluctuations and both the effects are included here. The results are shown in figure 2 .

The correlation $w\left(\nu_{1}, \nu_{2}, \theta\right)$ has the imprint of the large scale structures at high z. This signal might be swamped by galactic or extragalactic sources but it is hoped that the cross-correlation between different frequencies will help extract the desired signal. Work is underway in investigating these issues.

\section{References}

Lanzetta, K. M., Wolfe, A. M., Turnshek, D. A. 1995, ApJ, 430, 435

Swarup, G., Ananthakrishan, S., Kapahi, V. K., Rao, A. P., Subrahmanya, C. R., \& Kulkarni, V. K. 1991, Curr. Sci., 60, 95

Efstathiou, G., Bond, J. R. \& White, S. D. M. 1992, MNRAS, 250, 1p 